\title{
On the Galactic Evolution of Bipolar Planetary Nebulae
}

\author{
S. Durand ${ }^{1}$, J. Köppen ${ }^{2} \&$ W. Maciel ${ }^{1}$ \\ ${ }^{1}$ Instituto Astronômico da USP, São Paulo, Brazil \\ ${ }^{2}$ Observatoire Astronomique de Strasbourg, Strasbourg, France
}

Bipolar Planetary Nebulae (hereafter PNe) are almost all of the Type I and have the most massive progenitors among PNe. They are mostly found at very low galactic latitudes and thus are expected to follow rather closely the differential rotation. Moreover since they are strongly suspected to constitute the youngest population among $\mathrm{PNe}$, then some bipolars may have not achieved a complete galactic revolution yet and we can reasonably suppose that some signs of their recent galactic evolution still exist.

With this idea in mind we decided to investigate the kinematic properties of the 100 known galactic bipolars. We compiled various data associated with these objects, mainly radial velocities, statistical distances and chemical data.

Moreover, in order to increase the kinematic sample, we observed recently 9 bipolar PNe with the $1.60 \mathrm{~m}$ telescope equipped with the Coude Spectrograph (Observatório do Pico dos Dias, Brazil). The He II $\lambda 4686, \mathrm{H} \beta$ and [O III] lines were observed at medium resolution, allowing the estimation of the radial (lineof-sight) velocities with measurement uncertainties of $\simeq 13 \mathrm{~km} \mathrm{~s}^{-1}$.

The $(l, V)$ diagram of bipolar PNe shows they tend to lie along spiral features; a strong stellar clumping is observed along the Norma spiral arm's tangential view $\left(l \simeq 320^{\circ}\right)$. Bipolars are probably not very far from their formation sites.

We have detected a correlation between the dereddened $\mathrm{HeII} \lambda 4686 / \mathrm{H} \beta$ fluxes ratios of bipolar $\mathrm{PNe}$ and their galactocentric distances (derived from the hypothesis of a flat rotation curve) ; with the statistical distances the effect is less visible but also present. Bipolars with a high $\mathrm{He} \mathrm{II} / \mathrm{H} \beta$ ratio seem to deviate only slightly from the circularity. By contrast, stars with a low $\mathrm{He} \mathrm{II} / \mathrm{H} \beta$ ratio tend to be more highly dispersed in the galactic disk, probably because of an increase of the velocity dispersion with age. Thus it seems possible to retrieve the birth location of the stars and to better assess the diffusion of their stellar orbits.

Acknowledgments. This work was supported from Fapesp grant 99/070036 and $\mathrm{CNPq}$ 